\title{
Numerical Simulation of Quantum Many-Body Systems
}

DOE/ER/45197--T1

\section{Review of Work}

DE93 008300

\section{Hubbard model}

Results for the single-particle density of ștates and the conductivity were obtained for both the attractive- and repulsive- $U$ Hubbard models $[1,2] . *$ At half-filling the density of states for both models are identical, but the gap for the attractive case arises from the formation of charge-density-wave and superconducting correlations while for the repulsive$U$ Hubbard model the gap is the Mott-Hubbard gap and arises from the antiferromagnetic, Coulomb, correlations. When the attractive Hubbard model is doped away from half-filling, the Fermi level moves gradually away from the half-filled position, staying in the center of the gap. When the repulsive case is doped away from half-filling, we find that the chemical potential shifts to the top of the "lower Hubbard band" if electrons are removed or to the bottom of the "upper Hubbard band" if electrons are added. Results for the conductivity and the Drude weight as well as the susceptibility and the effective electronelectron interaction are contaired in these papers.

Hubbard chains were studied $[4,5]$ using a generalization of Handscomb's quantum Monte Carlo scheme. The ranges of validity of recently published asymptotic forms of spin correlation functions and susceptibilities were established. In addition, the finitetemperature crossover between talf-filled and non-half-filled behavior at low doping was examined.

Monte Carlo calculations of the two-particle vertex of the 2D repulsive- $U$ Bubbard model were carried out. Combining this with Monte Carlo results for the single-particle

- The refreaces in brackets are to the papers listed in Sec. III, Publications. 
propagator, we have determined the eigenvalues and eigenfunctions of the particle-hole and particle-particle Bethe-Salpeter equations for an $8 \times 8$ half-filled Hubbard lattice with $l^{*} t=$ 4 and $U / t=8[8]$. In the particle-hole channel, the dominant eigenvalue corresponded to the $\boldsymbol{Q}=(\pi, \pi)$ antiferromagnetic correlations. In the particle-particle channel the amplitude of the leading low-temperature eigenvalue was an everinfrequency $d_{x^{2}-y^{2}}$ singlet. Odd-frequency $p$-wave singlet and $g$-wave triplet anplitudes were also found.

An overview of our Monte Carlo work on the 2D Hubbard model was given this past summer at the Enrico Fermi Summer School [7], and the manuscript for the proceedings is at tached.

\section{Criteria for determining whether a system is insulating, metallic, or superconducting}

During the past year we investigated the criteria for determining whether a system is insulating, metallic, or superconducting $[3,6]$. For lattice models (Hubbard, Holstein, etc.) we showed that Monte Carlo calculations of the current-current correlation function

$$
\Lambda_{x x}\left(g, \omega_{m}\right)=\int_{0}^{\beta} d \tau e^{i \omega_{m} \tau}\left\langle j_{x}(q, \tau) j_{x}^{\dagger}(q, 0)\right\rangle
$$

with

$$
j_{x}(q)=-i e t \sum_{l} e^{i q l}\left(c_{l+\dot{x} s}^{\dagger} c_{l s}-c_{l s}^{\dagger} c_{l+x s}\right)
$$

provided with key. A system is insulating if in the limit of $T \rightarrow 0$ and linear dimension goes to $\infty$

$$
\Lambda_{x x}\left(q=0, \omega_{m} \rightarrow 0\right)=-\left\langle K_{x}\right\rangle
$$

Here $\left\langle K_{x}\right\rangle$ is the kinetic energy per site associated with bonds in the $x$-direction. It is metallic if

$$
\Lambda_{x x}\left(q=0, \omega_{m} \rightarrow 0\right)<-\left\langle K_{x}\right\rangle
$$

It is superconducting if

$$
\Lambda_{x z}\left(q_{x}=0, q_{y} \rightarrow 0, w_{m}=0\right\rangle<-\left\langle K_{x}\right\rangle \text {, }
$$


and gauge invariance implies that

$$
\Lambda_{x x}\left(q_{x} \rightarrow 0, q_{y}=0, \omega_{m}=0\right)=-\left\langle K_{x}\right\rangle .
$$

This work is important because it provides a specific way of determining the conducting state of a system. Thus one can investigate whether a system is superconducting without having to guess the symmetry of its order parameter. This work also clarified the limiting procedures necessary to distinguish the difference between the metallic and superconducting state in dimension greater than or equal to 2.

\section{Proposed Research}

As noted in the June DOE review, our project goal is to determine and understand the physical properties of models of strongly interacting many-electron systems, such as the Hubbard model, the Holstein electron-phonon model, and the Kondolattice. Our approach is to develop systematic calculational techniques: Lanczos, quantum Monte Carlo, summation of selected Feynman graphs. We are presently.working on the following projects.

1. Calculations of the two-particle vertex for the $\mathrm{CuO}_{2}$ model. We plan to examine both the particle-hole and particle-particle Bethe-Salpeter equations. We are particularly interested in examining the region of parameter space near the charge-transfer metalinsulator regime. It is possible that in this regime the two-particle vertex will have significant eigenvalues in the particle-particle singlet extended s-wave channel. Some evidence for this is provided by our earlier studies, ${ }^{1}$ which showed that the extended s-wave pairfield susceptibility was enhanced by the interactions. Now, with the ability to examine the Bethe-Salpeter equation, we can study this possible mode of pairing in much more detail.

2. The Kondo Lattice. Recent experimental transport measurements ${ }^{2}$ and neutron scattering ${ }^{3}$ results for CeNiSn raise many new questions about the Kondo lattice problem. 
We are planning further . Ionte Carlo studies of the Kondo lattice, ${ }^{4}$ in which the magnetic structure factor of the "localized f-electron" spins as well as the total magnetic structure factor will be examined as a function of temperature. In addition, the effect of an external magnetic field on the charge and spin gaps of the symmetric (half-filled) Kondo lattice will be determined.

3. Algorithms. An important part of our work has been the development of new techniques for solving quantum many-body problems. We are continuing our work on the development of algorithms for quantum Monte Carlo calculations with particular focus on the "fermion determinantal sign problem." For some models which we seek to study, the measure of the functional integrals is not positivie definite. In these models there are important parameter regions in which cancellations between the contributions of positive and negative weight make numerical calculations extremely difficult. We are investigating a number of approaches to this problem. We believe that modest improvements can be made by making use of more sophisticated data analysis techniques based on the jackknife and bootstrap methods. We plan to investigate whether the sign problem can be ameliorated by focusing on states in the immediately vicinity of the fermi surface, which make the main contributions to physical processes. Finally, we plan to determine whether a modified form of multicanonical algorithms, which have recently been used with some success in the study of spin models and lattice gauge theory, can be useful in our problems.

\section{References}

1. Antiferromagnetic, Charge-Transfer, and Pairing Correlations in the 3-Band Hubbard Model, R.T. Scalettar, D.J. Scalapino, R.L. Sugar, and S.R. White, Phys. Rev. B 44, 770 (1991).

2. T.E. Mason, et al., Phys. Rev. Lett. 60, 490 (1992).

3. G. Aeppli, et al., preprint.

4. One-Dimensional Symmetric Kondo Lattice: A Quantum Monte Carlo Study, R.M. Fye and D.J. Scalapino, Phys. Rev. Lett. 65, 3177-3180 (1990); Quantum Monte Carlo Study of the One-Dimensional Symmetric Kondo Lattice, R.M. Fye and D.J. Scalapino, Phys. Rev. B 44, 7486-7498 (1991). 


\section{Publications}

The following publications have resulted from DOE grant DE-FG03-85ER+519T

1. Quasi-Particle Gap in a Two-Dimensional Kosterlitz-Thouless Superconductor, A. Moreo. D.J. Scalapino, and S.R. White. Phys. Rev. B 45, 7544-7546 (1992).

2. Numerical Simulations of the Two-Dimensional Hubbard Mgdel: Dynamic Properties, D.J. Scalapino, Physica C 185-189. 104-113 (1991).

3. Superfluid Density and the Drude Weight of the Hubbard Model, D.J. Scalapino, S.R. White, and S.C. Zhang, Phys. Rev. Lett. 68, 2830-2833 (1992).

4. A Generalization of Handscomb's Quantum Monte Carlo Scheme-Application to the ID Hubbard model, A.W. Sandrik, J. Phys. A: Math. Gen. 25, 3667 (1992).

The following papers, preapred under this grant, have been submitted for publication.

5. Quantum Monte Carlo Study of Correlation Functions in the 1D Hubbard Model, A.W. Sandvik, D.J. Scalapino, anc C. Singh.

6. Insulator, Metal, or Superconductor: The Criteria, D.J. Scalapino, S.R. White, and S. Zhang, preprint UCSBTH-92-33, submitted to Phys. Rev. B.

7. Does The Hubbard Model Have the Right Stuff?, D.J. Scalapino, to appear in the Proceedings of the Enrico Fermi Summer School, Varenna, 1992.

8. Bethe-Salpeter Eigenvalues and Amplitudes for the Two-Dimensional Hubbard Model, N. Bulut, D.J. Scalapino, and S.R. White.

\section{Personnel}

In addition to the Principal Investigators, the personnel receiving support from this grant are:

Graduate student: A. Sandvik

Visiting faculty: D. Toussaint

In addition, N. Bulut, supported by an IBM. Postdoctoral Fellowship, has worked on this project.

\section{DISCLAIMER}

This report was prepared as an account of work sponsored by an agency of the United States Government. Neither the United States Government nor any agency thereof, nor any of their employees, makes any warranty, express or implied, or assumes any legal liability or responsibility for the accuracy, completeness, or usefulness of any information, apparatus, product, or process disclused, or represents that its use would not infringe privately owned rights. Reference herein to any specific commercial product, process, or service by trade name, trademark, manufacturer, or otherwise does not necessarily constitute or imply its endorsement, recommanufacturer, or otherwise does not necessarily constitute or inger States Government or any agency thereof. The views mendation, or favoring by the United state or reflect those of the and opinions of authors expites Government or any agency thereof. 

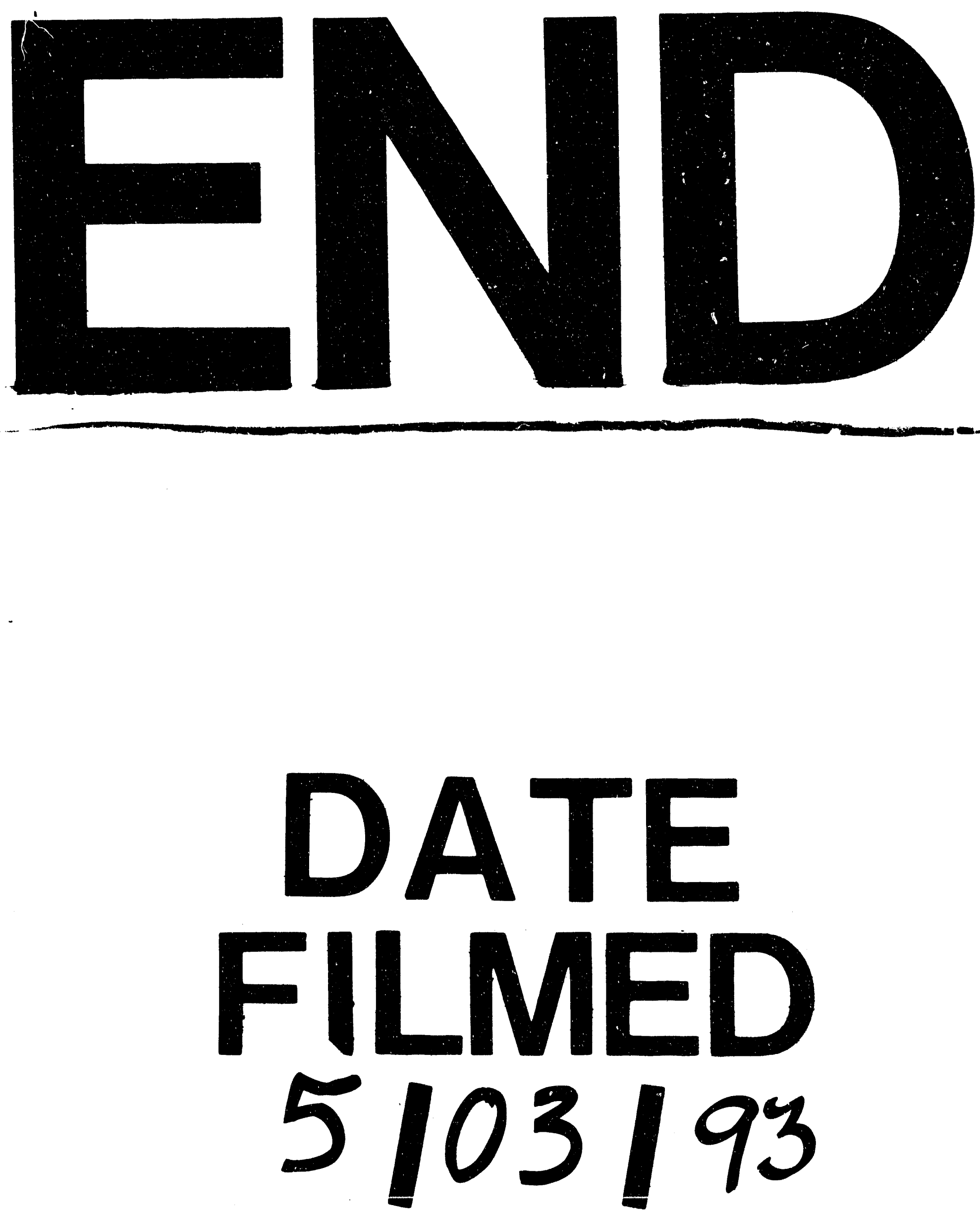
1 